\title{
Comment on "Balance recovery control for biped robot based on reaction null space method" by Baoping Wang, Renxi Hu, Jinming Zhang and Chuangfeng Huai
}

Dear Editor,

I am coauthor of the paper entitled "Balance control of a humanoid robot based on the reaction null space method" by Akinori Nishio, Kentaro Takahashi and Dragomir N. Nenchev, published on pages 1996 - 2001 in the Proceeding of the 2006 IEEE/RSJ International Conference on Intelligent Robots and Systems held 9-15 October 2006 in Beijing, China.

I recently found out that large portions of the above paper including whole paragraphs, some of the figures as well as part of the experimental results have been directly copied and published in the Journal of Control Theory and Applications of Springer, in a paper entitled "Balance recovery control for biped robot based on reaction null space method" by Baoping Wang, Renxi Hu, Jinming Zhang and Chuangfeng Huai, in Volume 7, Number 1, pages 87 - 91.

I believe that this is in violation of publishing ethics standards and can be definitely considered as a case of plagiarism. I believe also that the Journal of Control Theory and Applications, as well as the Publisher, aim to maintain high publishing ethics standards and hope therefore that appropriate steps will be undertaken to acknowledge the above fact in publicity both by the Journal/Publisher and the Authors, and also to prevent similar occurrences of plagiarism in the future.

Sincerely yours,

Dragomir N. Nenchev (Yoshikazu Kanamiya)

Tokyo City University

Tamazutsumi 1-28-1, Setagaya-ku, Tokyo

\section{Notification}

We hereby acknowledge that the published paper on our journal, "Balance recovery control for biped robot based on reaction null space method" by Baoping Wang, Renxi Hu, Jinming Zhang and Chuangfeng Huai, is a plagiarism of "Balance control of a humanoid robot based on the reaction null space method" by Akinori Nishio, Kentaro Takahashi and Dragomir N. Nenchev.

We withdrew the plagiarized paper from the Springer website, and sent a formal notification letter to each author's affiliation. Any submission from Baoping Wang, Renxi Hu, Jinming Zhang or Chuangfeng Huai will not be accepted in three years from now on. The above comment and this notification are published not only in this printed version, but also on the Springer website and our journal website.

We have used plagiarism scanning software and started the online detection of plagiarism in the received papers in September 2010 for screening the plagiarized submission.

Journal of Control Theory and Applications

October 28, 2010 